\title{
Parental compliance with home cardiorespiratory monitoring
}

\author{
T Carbone, B M Ostfeld, D Gutter, T Hegyi
}

\begin{abstract}
Aims-To evaluate parental compliance with home cardiorespiratory monitoring of premature infants with apnoea, siblings of infants who died of sudden infant death syndrome (SIDS), and infants with an apparent life threatening event (ALTE), during the first month of use.
\end{abstract}

Methods-A retrospective review of the first month's recordings was conducted on 39 premature infants with apnoea, 13 siblings of SIDS, and 16 infants with ALTE. All infants were singletons. Recommendations during the study period (1992-1994) were for daily use for 23 hours per day. Measurements were average daily hours of use and consistency of use (daily or variable). Gestational age, maternal age, and socioeconomic status as measured by receipt of public assistance were also recorded.

Results-Siblings of SIDS were monitored for fewer hours than were premature or ALTE infants. Only $54 \%$ of sibings of SIDS were monitored daily, compared to $87 \%$ of premature infants and $93 \%$ of ALTEs. Within each diagnostic category socioeconomic status did not affect average hours of monitoring. Consistency of use was more evident in those with private insurance, although the trend did not reach significance.

Conclusions-Parents of infants with apnoea of prematurity or ALTE are highly compliant with cardiorespiratory monitoring recommendations in the first month of monitor usage. Siblings of SIDS are monitored for fewer hours and are less likely to be monitored on a daily basis. (Arch Dis Child 2001;84:270-272)

Keywords: cardiorespiratory monitoring; apnoea; prematurity; apparent life threatening event; SIDS

Division of

Neonatology,

UMDNJ-Robert Wood

Johnson Medical

School, St Peter's

University Hospital,

New Brunswick, NJ

08903, USA

T Carbone

B M Ostfeld

D Gutter

T Hegyi

Correspondence to:

Dr T Carbone, The Valley

Hospital, 223 North Van

Dien Avenue, Ridgewood, NJ

07450, USA

tcarbon@valleyhealth.com

Accepted 21 September 2000 programmes. However, of the few studies that have addressed this issue by examining compliance with respect to diagnostic categories, none has provided a specific and standard directive of daily hours to be achieved. ${ }^{1-3}$ Thus, it is dif- ficult to interpret whether variations in monitor usage reflect problems with compliance issues or individual differences in the child care practices that are referenced in non-specific monitoring guidelines.

The objective of our study was to determine care giver compliance with specific and standard directives for daily home monitoring based on the diagnostic indications for use.

\section{Methods}

STUDY POPULATION

Candidates consisted of singleton infants for whom monitoring was recommended for one of the following indications: preterm birth (birth weight less than $1500 \mathrm{~g}$ ) with unresolved apnoea of prematurity, idiopathic apparent life threatening events, or the death of a sibling from SIDS. Candidates were enrolled in the infant apnoea clinic between October 1992 and June 1994, had a corrected age of 2 months or less, and came from families in which the primary language spoken at home was English.

\section{PROCEDURE}

A retrospective chart review of the first month's cardiorespiratory monitor recordings was conducted on all singleton subjects in a study of monitor usage. Parents of eligible infants were approached during the infant apnoea clinic visit that followed the first routine download. Downloads were performed routinely on all patients on a monthly basis prior to each patient visit. Recruitment was conducted only during those sessions when an investigator was available in the waiting room. Ninety seven per cent of those approached agreed to enrol. The approval of the institutional review board was obtained.

At the initiation of monitoring, all mothers were routinely instructed by the infant apnoea programme coordinators to use the monitor 23 hours daily. The programme provided ongoing evaluation, education, training, medical follow up, and psychosocial support. Documented monitors (Healthdyne 970S), which record transthoracic impedance and electrocardiogram signals, were used. These monitors record the day and time of use and display this information graphically. Monitor use was summarised by calendar days in hours and minutes. The monitor recordings that are the focus of the present study summarise the first month of monitor use and represent a period that precedes the invitation to parents to participate in this study.

\section{MEASURES}

Two measures were used for compliance. The measure for average daily hours of use was 
Table 1 Subject characteristics of monitored premature, siblings of SIDS, and ALTE groups

\begin{tabular}{llll}
\hline Variable & Premature group & Siblings of SIDS & ALTE group \\
\hline $\mathrm{N}$ & 39 & 13 & 16 \\
Gestational age (wk) $\dagger$ & $30.2(2.6)^{\star}$ & $37.4(3.6)$ & $38.8(2.0)$ \\
Maternal age (y) $\dagger$ & $30.2(4.9)$ & $28.0(6.8)$ & $32.0(6.4)$ \\
Public assistance & $9(23 \%)$ & $8(62 \%)^{\star}$ & $2(13 \%)$ \\
\hline
\end{tabular}

†Data expressed as mean (SD).

${ }^{\star} \mathrm{p}<0.001$.

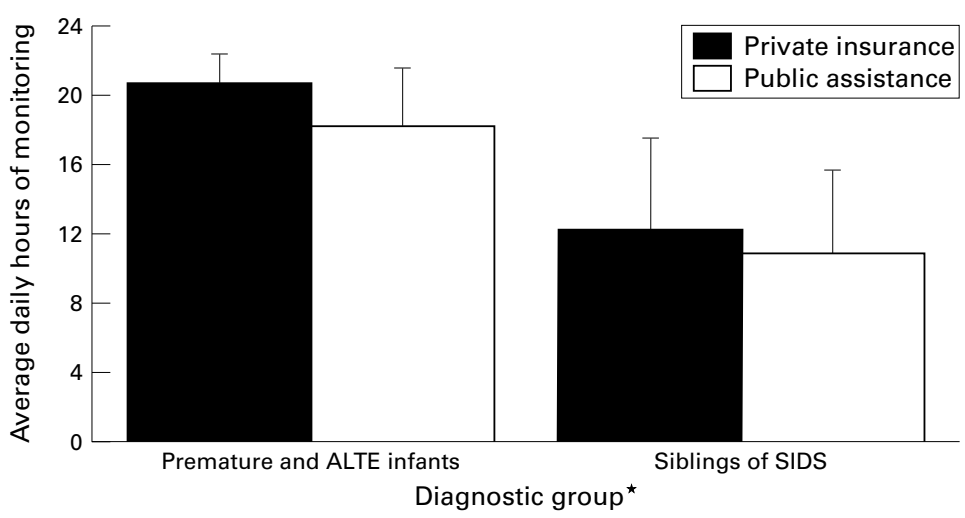

Figure 1 Average daily hours of cardiorespiratory monitoring, on days used, by diagnosis and socioeconomic status. ${ }^{\star}$ Diagnostic group effect: $F(1,64)=35.8, p<0.0001$.

based on all calendar days ( 24 hour periods) on which the monitor was used. The measure for variability of use was determined by categorising each case into daily use (regardless of hours) or less than daily use.

STATISTICAL METHODS

An analysis of variance (ANOVA) was used to compare the average daily hours of use scores as well as the gestational ages and maternal ages of the premature, siblings of SIDS, and apparent life threatening event (ALTE) groups. A two by two ANOVA was used to evaluate the interaction of diagnostic group and socioeconomic status on the average daily hours of use scores. Fisher exact or $\chi^{2}$ tests were used for the categorical data of daily versus intermittent monitor use and socioeconomic status (public assistance versus private insurance). Two tailed $t$ tests were used to compare the average daily hours of use scores of daily versus intermittent users of monitors.

\section{Results}

Table 1 presents demographic data. Maternal age was comparable in the premature, siblings of SIDS, and ALTE groups. By definition, the average gestational age of the premature group was lower than that for the siblings of SIDS and ALTE groups, both of which were full term $(F(2,65)=7.8, p<0.001)$. Significantly more siblings of SIDS were on public assistance $\left(\chi^{2}(2)=9.6, \mathrm{p}<0.01\right)$.

Siblings of SIDS were monitored for fewer hours a day than were the other groups

Table 2 Daily versus variable use of cardiorespiratory monitoring by socioeconomic status

\begin{tabular}{llll}
\hline & \multicolumn{2}{l}{ Daily use } & \\
\cline { 2 - 3 } & Public assistance & Private insurance & p value \\
\hline Premature and ALTE infants & $8 / 11(73 \%)$ & $41 / 44(93 \%)$ & $<0.06$ \\
Siblings of SIDS & $3 / 8(37 \%)$ & $4 / 5(80 \%)$ & \\
\hline
\end{tabular}

$(F(2,65)=24.6, p<0.0001)$. For siblings of SIDS, usage was 11.4 (6.1) hours, in contrast to premature and ALTE infants who scored at 20.0 (3.5) hours and 20.2 (3.1) hours, respectively. Because the monitor rates for the latter two groups were comparable, they were combined for further analysis.

Figure 1 presents the effect of socioeconomic status on diagnostic group with respect to monitor hours. No effect was found within either diagnostic group. The 44 premature infant and ALTE group cases with private insurance were monitored for 20.6 (2.9) hours and the 11 cases with public assistance were monitored for 18.2 (4.4) hours. In the sibling of SIDS group, the five privately insured subjects were monitored for 12.3 (6.6) hours and those with public assistance for 10.9 (6.2) hours.

All three diagnostic groups were also evaluated with a measure of variability, daily versus less than daily monitor use. The siblings of SIDS group was least likely to be monitored on a daily basis, $\chi^{2}(2)=9.3, p<0.01$, reinforcing the compliance patterns obtained for average daily hours of monitoring analysis. In contrast to the premature group and the ALTE group, in which daily compliance was achieved by 34 of $39(87 \%)$ and 15 of $16(93 \%)$ of the cases, respectively, only seven of $13(54 \%)$ of the siblings of SIDS cases were so monitored. Because the monitor rates for the former two groups were again comparable, they were combined for further non-parametric analysis.

Table 2 presents the effect of socioeconomic status on the consistency of monitor use for each diagnostic group. Within the combined premature and ALTE group, daily monitoring was achieved by more cases with private insurance than public assistance. This difference approached significance $(\mathrm{p}<0.06)$. For siblings of SIDS, daily monitoring was also more common for cases with private insurance than public assistance; however, this trend also failed to reach significance.

A relation was found between the two compliance measures: consistency of use (daily or less) and the average daily hours of monitoring. In the combined group of premature and ALTE patients, the 56 daily users monitored for 20.9 (2.4) hours, compared to 13.6 (3.0) average daily hours by the 12 parents who used the monitor less consistently $(t(53)=6.9$, $\mathrm{p}<0001)$. In the siblings of SIDS group, the seven daily users monitored for an average 15.6 (3.7) hours of daily use, compared to the six less consistent users who monitored for an average of 6.5 (4.3) hours $(t(11)=4.1$, $\mathrm{p}<0.01)$.

\section{Discussion}

In the first month following its prescription, parents of infants with apnoea of prematurity or ALTE are extremely compliant with recommendations for cardiorespiratory monitor usage. At approximately 20 hours of usage per day, apnoea and ALTE families exceeded the 15 hours of daily usage that Silvestri et al concluded were representative of excellent compliance. ${ }^{2}$ In contrast to these groups, families of 
SIDS siblings monitored for only half as many hours a day. This finding was in contrast to our clinical expectations which presumed that the historical event of an SIDS death would elevate monitor compliance in subsequent siblings.

Only $54 \%$ of SIDS siblings were monitored daily, showing a significantly less consistent usage pattern than that for infants with apnoea of prematurity or ALTE. This finding differed from that of Silvestri et al whose SIDS siblings group approximated the highly consistent usage findings of their ALTE group. ${ }^{2}$ However, as in the present study, their SIDS siblings were surpassed in consistency of usage by infants with apnoea of prematurity.

In keeping with the profile of SIDS risk factors, more SIDS siblings received public assistance. ${ }^{4}$ Therefore, we analysed the effect of this variable on compliance patterns. We substantiated the finding of Silvestri et al that hours of use were not significantly associated with insurance type. ${ }^{2}$ However, in our study, consistency of use was more likely to be found among privately insured families in all groups, with the combined premature and ALTE group result approaching a significant difference between socioeconomic levels. In the SIDS siblings group, the small sample size may account for the failure of the difference between socioeconomic groups to reach significance. Cordero et al also obtained more consistent monitoring of infants with apnoea of prematurity in subjects without public assistance. ${ }^{1}$

Although we attempted to compare usage patterns in the present study with other studies, there are variations in methodology. These methodological differences include a lack of uniformity in daily hourly requirements, ${ }^{1-3}$ diagnostic categories, ${ }^{15}$ and duration of the monitor period under study. ${ }^{3}$ For example, in place of a specific recommendation for daily hours of use, guidelines for monitoring were based on child care practices and infant arousal states with monitoring recommended during infant sleep or when the infant was not under direct surveillance. Given differences in feeding requirements for premature and term infants, as well as in the variety of care giving patterns or number of available care givers, optimal monitoring could therefore be achieved with a broad range of hours. By specifying 23 hours per day, our study addresses the lack of a uniform usage goal that was noted by Silvestri et $a .^{2}$

Although a specific daily time goal was not provided, Silvestri et al used comparable diagnostic groupings and limited the period of study to the first month's download, thereby providing a basis for comparison. ${ }^{2}$ Perhaps reflecting the absence of a 23 hour per day guideline, their groups only monitored for an average of 15.5 hours per day, possibly reducing the differences between groups. However, although their SIDS siblings group was not monitored for significantly fewer hours per day than were infants with apnoea of prematurity or apparent life threatening events, it did rank lowest of the three diagnostic categories with an average hourly rate that was virtually identical to that found in the present study.

Our study called for enrolment following the first month's download so that the retrospective information would be untainted by the placebo effect of being enrolled in a study. As a result, structured interviews with the families for the purpose of identifying factors that might reduce compliance were not conducted prior to or concurrent with the first month of documented home monitoring. Moreover, because the study was limited to the first month of use when high compliance provided not only therapeutic value but also diagnostic data, the families were advised to maintain compliance regardless of the rate of alarms, and compliance was not evaluated with respect to alarm frequency. Therefore, we can only speculate about the aetiology of the variance in compliance that is unaccounted for by socioeconomic factors, and we suggest that it may be a function of two as yet unexplored factors. Firstly, parents of premature and ALTE infants have observed their infants in a symptomatic state and have witnessed the cascade of medical reactions to these symptoms, while parents of SIDS siblings do not have such an identified patient. Although SIDS parents have experienced the most extreme crisis, the events are historical and may engender a different set of emotions and behaviours with respect to the asymptomatic subsequent sibling. For example, parents of SIDS siblings might be reluctant to perceive the subsequent child as vulnerable and take comfort in the low probability of a recurring SIDS diagnosis. Or, they may be quite concerned and therefore more likely to hold and directly observe the infant in lieu of monitoring. Secondly, we hypothesise that ongoing discussions over the efficacy of monitoring for SIDS may contribute to potentially conflicting recommendations with respect to siblings of SIDS in particular and that the resulting variance in approaches may adversely affect compliance. If these speculations are correct, SIDS families who are advised to monitor subsequent siblings may require additional education and family support, and more frequent follow up by the medical team.

This work was presented in part at the International Congress on SIDS, Graz, Austria, 26 May 1995, and the Eastern Society for Pediatric Research, Atlantic City, NJ, 1 March 1997.

1 Cordero L, Morehead S, Miller R. Parental compliance with home apnea monitoring. F Perinatol 1993;13:448-52.

2 Silvestri JM, Hufford DR, Durhan J, et al. Assessment of compliance with home cardiorespiratory monitoring in infants at risk of sudden infant death syndrome. F Pediatr 1995;127:384-8.

3 Cote A, Hum C, Brouillette RT, Themens M. Frequency and timing of recurrent events in infants using home cardiand timing of recurrent events in infants using ho

4 Hoffman HJ, Hillman LS. Epidemiology of the sudden infant death syndrome: maternal, neonatal and postneonatal risk factors. Clin Perinatol 1992;19:717-37.

5 Mohan CG, Baird TM, Super DM, et al. Weekly telephone contact does not enhance the compliance of home apnea monitoring. F Perinatol 1999;19:505-9. 